\title{
Somatosensory processing in neurodevelopmental disorders
}

\author{
Carissa J. Cascio
}

Received: 7 February 2010/Accepted: 7 April 2010/Published online: 20 April 2010

(C) Springer Science+Business Media, LLC 2010

\begin{abstract}
The purpose of this article is to review the role of somatosensory perception in typical development, its aberration in a range of neurodevelopmental disorders, and the potential relations between tactile processing abnormalities and central features of each disorder such as motor, communication, and social development. Neurodevelopmental disorders that represent a range of symptoms and etiologies, and for which multiple peer-reviewed articles on somatosensory differences have been published, were chosen to include in the review. Relevant studies in animal models, as well as conditions of early sensory deprivation, are also included. Somatosensory processing plays an important, yet often overlooked, role in typical development and is aberrant in various neurodevelopmental disorders. This is demonstrated in studies of behavior, sensory thresholds, neuroanatomy, and neurophysiology in samples of children with Fragile X syndrome, autism spectrum disorders (ASD), attention deficit hyperactivity disorder (ADHD), and cerebral palsy $(\mathrm{CP})$. Impaired somatosensory processing is found in a range of neurodevelopmental disorders and is associated with deficits in communication, motor ability, and social skills in these disorders. Given the central role of touch in early development, both experimental and clinical approaches should take into consideration the role of somatosensory processing in the etiology and treatment of neurodevelopmental disorders.
\end{abstract}

Keywords Touch $\cdot$ Development $\cdot$ Tactile $\cdot$ Sensory integration $\cdot$ Neurodevelopmental disorders

\section{J. Cascio $(\bowtie)$}

Vanderbilt Kennedy Center for Research on Human Development, Vanderbilt University Department of Psychiatry,

1601 23rd Avenue South, Suite 3057,

Nashville, TN 37212, USA

e-mail: carissa.cascio@vanderbilt.edu

\section{Introduction}

Somatosensory perception plays a central role in the early stages of human development. Among all sensory systems, the tactile sense develops earliest, and somatosensory responses can be detected in utero as early as 8 weeks gestational age (Montagu 1986), with cortical responses showing measurable maturation during the preterm-term period (Pihko and Lauronen 2004). Neonates immediately make use of perioral tactile input that elicits rooting and sucking reflexes for nursing (Muir 2002). Throughout infancy and early childhood, the sense of touch provides important information and feedback about the child and its world, influencing the development of motor, social, and communication skills.

The development of both gross and fine motor skills depends heavily on the somatosensory system. Infants initially depend on touch cues to initiate reaching for objects, and later use vision as well (Corbetta and SnapChilds 2009). Accurate tactile and proprioceptive sensation is critical for the development of motor skills during childhood, with somasthetic touch being particularly important for fine motor development (Cauller 1995) during the preschool years (Case-Smith 1995). Tactile feedback from mechanoreceptors in the skin and joints critically guides the online modulation of gross motor functions such as walking (Metcalfe et al. 2005), and fine motor functions such as grasping (Soechting and Flanders 2008), during early development and throughout the lifespan.

Although the role of somatosensory input for motor development is well established and transparent, touch is also critical for healthy social and communication skills in early childhood and beyond. Somatosensory input from maternal contact during feeding and ventral-ventral contact 
contributes to an ongoing association of caregivers with comfort and nourishment, facilitating the infant's adaptation to its novel environment (Porter 2004). This tactile associative learning is critical for the establishment of bonding and secure attachment (Main and Stadtman 1981; Myers 1984; Weiss et al. 2000), which lay the foundation for the development of healthy social behaviors throughout the lifespan. In many cultures, extensive tactile contact with parents and older siblings is a means of establishing a secure base from which young children venture to explore their environment (Sorensen 1979).

During the significant period of time prior to the acquisition of verbal language, touch is a primary mode of communication between babies and caregivers (Montagu 1986). Touching behaviors in mothers communicate reassurance (Jean and Stack 2009), affection, and can be used to direct attention (Ferber et al. 2008). Higher levels of affectionate maternal touch in infancy result in increased infant smiling and vocalization (Stack and Muir 1992), and frequency of maternal touch is associated with mother-infant reciprocity and the coordinated timing of interactions between mother and infant (Ferber et al. 2008). These reciprocal rhythms of interaction learned in infancy lay the foundation for social communication later in life. Infants also use touch to communicate distress (Moszkowski et al. 2009) and basic needs. Thus, tactile communication serves as a precursor (Dunbar 1996) to verbal communication, and the patterns formed during this period impact the development of more sophisticated communication skills as well as a framework for interaction with family and community. Even in adults, tactile input can enhance perception of auditory and visual speech (Gick et al. 2008), and is a primary means of nonverbal communication.

While the direct use of interpersonal touch is an important nonverbal communication method, other nonverbal communication skills such as gestures and facial expression are also dependent on the somatosensory system, via proprioceptive feedback from the hands and arms, as well as stretch receptors in facial skin and muscles. These skills are critical for the development of more subtle communication during the lifespan.

Hertenstein and colleagues (2006) point out that, in addition to the ontogenetic primacy of touch for social communication in early development, touch also has phylogenetic primacy for communication. In the evolutionary history of primate species, nonverbal communication, such as tactile interactions during grooming behaviors, preceded verbal communication. Dunbar (1996) suggests that only as hominids evolved to form larger groups and forage in larger territories did the need for a distal modality of communication emerge, selecting for the vocalizations that led to verbal communication. Thus, the human tactile sense has, at its evolutionary roots, a central role for communication, and is the precursor to verbal communication.

Neurodevelopmental disorders (NDDs) comprise a broad range of conditions that emerge in childhood and for which known or presumed aberrations in nervous system function give rise to behavioral, social, emotional, motor, or cognitive differences. As reviewed above, touch plays a prominent role in each of these areas, especially during the early stages of their development. This review will focus on a subset of NDDs for which there is a primary area of impairment in one or more of these areas, and for which there is substantial converging data on somatosensory processing from behavioral, psychophysical, and neurobiological approaches. NDDs chosen for inclusion in this review also represent a range of etiologies, from a known mutation on a single gene (Fragile $\mathrm{X}$ syndrome), to disorders with polygenetic or unknown genetic origins (ADHD and ASD), to disorders arising primarily from perinatal or early environmental insult (CP and early sensory deprivation). Behavioral activity level and attention is affected in attention deficit-hyperactivity disorder (ADHD), and several studies have explored somatosensory processing in this group. Children with autism spectrum disorders (ASD) suffer from impaired social and communication skills, and sensory processing difficulties, including tactile defensiveness, are a common associated feature. In contrast to these genetically complex, behaviorally defined disorders, Fragile $\mathrm{X}$ syndrome arises from a mutation of the gene that codes for the FMR1 protein, which has a key role in early brain development. Symptoms of Fragile X include mild intellectual disability, low muscle tone, hyperactivity, and hypersensitivity to sensory stimuli. There is also some overlap between symptoms of ASD and Fragile X. Cerebral palsy (CP) is a group of nonprogressive disorders primarily affecting motor development that arises from insult or injury in the prenatal or perinatal environment. The motor deficits seen in $\mathrm{CP}$ are primarily abnormalities in muscle tone, and are associated with widespread differences in somatosensory perception as well (Clayton et al. 2003).

Similarly, early environmental conditions that are isolating or impoverished result in profound developmental problems in behavior, emotion, cognition, and sensory perception. While not a separate diagnostic classification, children for whom this applies do have severely disordered neural development and provide an illustrative example of the importance of somatosensory input early in development, and will be discussed in the conclusion of this review.

The neural substrates of touch perception are among the most carefully studied and thoroughly understood systems in the brain, as well as among the earliest to develop. Yet, in the investigation of NDDs, tactile processing is often overlooked as a variable of interest in favor of far more 
complex cognitive, verbal communication and social abilities, most of which fully develop long after the window in which brain changes are known to give rise to NDDs. Thus, a review of the existing literature examining tactile processing in NDDs is important to summarize our knowledge of the role of touch in NDDs, and to serve as a springboard for further investigation of this important but neglected area. Somatosensory processing in NDDs has been studied using behavioral measures such as observational assessment and parental report, classical psychophysical methods to determine thresholds, neuroanatomical/ neurophysiological methods including somatosensory evoked potentials (SEP) and magnetic resonance imaging (MRI) techniques, and animal models.

\section{Behavioral measures of somatosensory processing}

Clinicians, behaviorists, and developmental psychologists have pursued the characterization of tactile responsiveness in NDDs, employing direct observational assessment and parent questionnaires as techniques. Among other sensory symptoms, tactile defensiveness is a very common associated feature of ASD and Fragile $\mathrm{X}$ syndrome. Tactile defensiveness is defined as a hyperreactive and/or aversive response to tactile stimuli that most people would consider innocuous, such as light touch or clothing texture. Tactile defensiveness may also be at the root of idiosyncratic eating habits, as the texture or mouth-feel of many foods are perceived as aversive (Harris 2008; Field et al. 2003). Evidence from parent questionnaires suggests that tactile defensiveness is more common in Fragile $\mathrm{X}$ syndrome and ASD than in typical development (Tomchek and Dunn 2007), with rates slightly higher in Fragile $X$ syndrome (Baranek et al. 2008; Rogers et al. 2003). The relation between tactile defensiveness and the full complement of core features of either disorder remains unclear, but tactile defensiveness is significantly associated with certain kinds of rigid and stereotyped behaviors in ASD and other developmental disabilities (Baranek et al. 1997). In ADHD, sensory hypersensitivities are associated with level of hyperactivity (Yochman et al. 2004), and tactile defensiveness specifically may relate to genetic susceptibility for ADHD (Scherder et al. 2008) and comorbid anxiety (Reynolds and Lane 2009) and behavior problems (Ghanizadeh 2008). Tactile defensiveness also appears to interact with gender in this population, as Bröring et al. (2008) found that girls with ADHD tended to show higher levels of tactile defensiveness than boys with ADHD. Sleep and behavior problems in typically developing children are significantly associated with tactile hypersensitivity (Shochat et al. 2009).
Unusual responses to tactile stimulation in NDDs are not limited to tactile defensiveness. While tactile defensiveness is associated with aversive reactions, tactile "seeking" behaviors result when typically neutral tactile stimuli are perceived as highly pleasurable. Tactile seeking is common in ASD and can manifest as repetitive rubbing of certain textures or surfaces, or an affinity for deep pressure input such as intense hugging or squeezing (see Grandin 2000). Experimental studies also provide evidence of pleasurable responses to specific tactile stimuli (Pernon et al. 2007). Children with CP tend to seek out hard versus soft objects, which may be a manifestation of sensory seeking resulting from decreased somatosensory awareness (Curry and Exner 1988).

\section{Psychophysical measures somatosensory processing}

It might appear obvious that individuals who show tactile defensiveness, such as children with ADHD, Fragile X, and ASD, have heightened perceptual ability that would translate into increased performance in basic psychophysical sensory threshold measurements. Threshold testing depends on systematic stimulus-response paradigms to determine the smallest amount of signal (such as the smallest amplitude of a vibration, or the smallest difference in temperature) that is reliably perceptible by the individual. However, Parush et al. (1997) tested a sample of children with ADHD with tactile defensiveness and found that their tactile thresholds were normal, suggesting that tactile defensiveness may represent more of a cognitive or affective aberration than a sensory one in this sample.

In contrast, enhanced basic sensory perception in people with ASD has been supported by several studies (reviewed in Mottron et al. 2006), and has been noted in the tactile system specifically (O'Riordan and Passetti 2006; Blakemore et al. 2006; Cascio et al. 2008). Blakemore and colleagues found that adults with Asperger syndrome had heightened sensitivity to certain frequencies of vibration. A subsequent study failed to replicate high frequency vibrotactile thres hold differences at the fingertip in children with ASD (Güçlü et al. 2007), but was limited by a small sample size, which may have reduced power to detect group differences if present. The authors of this study did, however, note significant correlations between tactile and affective items on parent questionnaires, suggesting that aberrant responses to tactile stimulation may arise from cognitive and emotional mechanisms, rather than from differences in physiological response of the somatosensory system.

The possibility that tactile defensiveness in ASD is at least partially modulated by affective neural systems of touch as opposed to discriminative touch pathways is supported by a study from Cascio and colleagues (2008) 
in which adults with autism were tested on a variety of psychophysical tasks, including vibrotactile detection, light touch detection, thermal pain thresholds, and texture pleasantness estimations. In this study, both the palmar surface and the dorsal forearm were tested. In addition to extending tactile psychophysics beyond the fingertip, an advantage of testing the forearm site is its innervation by a class of peripheral afferents known as C-touch afferents, which are believed to constitute a unique system for conveying affective touch signals (Liu et al. 2007; Löken et al. 2009; McGlone et al. 2007; Olausson et al. 2002), and which do not innervate the palmar surface of the hand (Vallbo et al. 1999). Group differences that were specific to the forearm and greater sensitivity for painful thermal stimuli (with normal thresholds for innocuous thermal stimuli) suggest a particular abnormality in affective touch systems. While psychophysical studies of discriminative touch processing in ASD have yielded mixed results, the emotional aspects of touch are more consistently affected in ASD (Cascio et al. 2008; Güçlü et al. 2007). Given the importance of affective touch in early social development, an important future direction for the field is the comparison of affective touch perception in ASD and other NDDs that do not directly impact social functioning.

There are ubiquitous reports of impaired discriminative tactile abilities in CP (see Clayton et al. (2003) for a comprehensive review), and these impairments are closely associated with motor difficulties (van Heest et al. 1993). Wingert et al. (2008) found texture perception to be impaired in a CP sample relative to controls. Impaired stereognosis (shape perception) has been replicated many times (Cooper et al. 1995; Van Heest et al. 1993; Wingert et al. 2008), although this skill is likely to be particularly affected by motor impairments in manipulating objects (Kunesch et al. 1989; Dahlin et al. 1998). Also impaired is finer-grained tactile spatial discrimination (Van Heest et al. 1993; Lesny et al. 1993; Sanger and Kukke 2007). The magnitude of these deficits is substantial in light of the relatively mildly affected motor abilities of some of the children in these samples (Wingert et al. 2008), and the scope of sensory impairment suggests that somatosensory processing impairments have significant functional relevance for motor deficits in $\mathrm{CP}$.

\section{Neuroanatomical and neurophysiological measures of somatosensory processing}

While classical psychophysical approaches such as detection threshold measurement may point to potential neural mechanisms for somatosensory deficits in NDDs, they may not effectively separate peripheral from cortical mechanisms, or give much information about neurophysiological mechanisms or neuroanatomical loci of differences. Prominent cortical models of ASD have informed less traditional psychophysical studies of somatosensory processing in this disorder. Tommerdahl et al. (2007) tested adults with autism on a paradigm in which vibrotactile localization is improved by exposure to a previous adapting stimulus, which tunes subsequent signals via GABA-mediated inhibition of neighboring cortical minicolumns. Adults with ASD exhibited the increased sensitivity in baseline performance that would be predicted by other studies demonstrating enhanced tactile perception. However, they did not exhibit the improvement in the presence of the adapting stimulus that the control group did, suggesting that cortical inhibitory mechanisms may not function normally. Further evidence for aberrant cortical processing comes from abnormal somatosensory evoked potentials (Miyazaki et al. 2007) and a magnetoencephalography (MEG) study of somatosensory cortex in autism, Coskun et al. (2009) demonstrated unusual organization of somatotopic maps representing the body surface in the brain.

The picture of the neural basis of altered somatosensory processing in ASD is a complex one that will require considerable additional study. The co-occurrence of tactile hyperresponsiveness/defensiveness and hyporesponsiveness/seeking presents a challenge to experimental design. Current evidence suggests that emotional touch is more affected than discriminative touch, and differences in discriminative ability tend to fall on the side of enhanced perception in ASD. There is also burgeoning evidence that organization of somatosensory cortex is aberrant in this group of NDDs.

Brain responses of children with ADHD to somatosensory stimuli are also unusual, reflecting behavioral evidence of their differences in tactile perception. Children with ADHD and tactile defensiveness exhibit significantly larger somatosensory evoked potential (SEP) amplitudes over central electrodes than either controls or ADHD children without tactile defensiveness (Parush et al. 2007). Primary and secondary somatosensory cortical responses to electrical stimulation of the median nerve were measured with MEG from adults with ADHD by Dockstader and colleagues (2008). They found altered patterns of synchronization and desynchronization in the ADHD group. Using a similar paradigm, this group investigated cortical oscillations in response to unpredictable median nerve stimulation (Dockstader et al. 2009). These rhythms are believed to represent a mechanism for linking perception and action, an ability that may be compromised in ADHD. The result of this study was that the ADHD group showed lower oscillatory activity relative to controls, suggesting a deficit in the perception-to-action system. Finally, a structural brain study using voxel-based morphometry conducted by Carmona et al. (2005) demonstrated reduced gray matter in 
a variety of brain regions including somatosensory cortex in ADHD. The centrality of tactile perception for motor planning and the role of affective touch in arousal levels may be important in understanding tactile dysfunction in ADHD.

Neural studies of somatosensory processing in CP have provided insight into the physiological basis for these sensory differences. Kulak et al. (2006) noted altered SEPs in a CP group in response to somatosensory stimulation. Tomita and colleagues (2006) noted that the amplitude and topographic distribution of SEPs varies across subtypes of $\mathrm{CP}$, which may reflect differential involvement of various sensorimotor systems. Investigation of sensory thalamocortical pathways by Hoon et al. (2009) using diffusion tensor imaging (DTI) demonstrated significant relationships between tactile thresholds and periventricular white matter injury that precipitated $\mathrm{CP}$, a significant finding in light of the traditional understanding that motor, not sensory tracts, are the primary site of damage in CP (Wilke and Staudt 2009). These results highlight the inextricable link between somatosensory and motor systems.

\section{Animal models of somatosensory processing}

In the pursuit of biological mechanisms of impairments in NDDs, animal models provide invaluable information that allow a closer look into the molecular and genetic processes that underlie symptoms, including impaired somatosensory processing.

Because it is associated with a predictable mutation on a single gene, animal models of Fragile $\mathrm{X}$ have been useful in understanding more about the neurobiology of the disorder. In strains of mice that are missing the FMR1 gene, profound effects are seen in the organization of somatosensory cortex. FMR 1 has a role in the development of sensory cortical circuits (Bureau et al. 2008) which may be manifested by FMRP's control of GABA-releasing interneurons (Selby et al. 2007) that are intrinsic to the functioning of cortical circuits, or by its role in the normal maturation of dendritic spines in somatosensory cortex (Galvez et al. 2003; Galvez and Greenough 2005; Greenough et al. 2001). Compensatory modulation of environmental sensory exposure can rescue both cortical and behavioral effects in mice with the Fragile X mutation. Interestingly, this has been achieved both with deprivation (Bureau et al. 2008) and enrichment (Restivo et al. 2005) of the sensory environment.

Animal models also allow the experimental investigation of touch deprivation in early development. Tactile deprivation in infant rats results in impaired active touch discriminative abilities (Carvell and Simons 1996) similar to its effect in humans (Cermak and
Daunhauer 1997; Lin et al. 2005). As in humans, however, the effects of reduced contact early in infancy in animal models extend well beyond sensory abilities (Harlow and Harlow 1962; Cermak and Groza 2004). Lack of tactile maternal contact (experimentally isolated from possible confounding variables such as deprivation of nourishment or heat) results in reduced levels of essential growth hormone gene expression (Schanberg et al. 2003). Main and Stadtman (1981) found that lack of ventral-ventral contact early in infancy is associated with repetitive behaviors such as echolalia and stereotypies. The animal literature is replete with studies linking abnormal or absent early maternal touch to decreased cognitive abilities, novelty exploration, and attachment (Hertenstein et al. 2006).

These studies from the animal literature have prompted exploration of touch-based intervention for isolated premature human infants with dramatic success (Schanberg and Field 1987; Kuhn and Schanberg 1998). A meta-analysis of studies of infant massage efficacy suggests that interactions between mother and infant are significantly improved by regular touch (Underdown et al. 2006) and additional touch has been demonstrated to improve developmental outcomes in institutionalized infants (Casler 1965). These studies emphasize the important role of touch in early development, and suggest that therapeutic approaches incorporating touch should continue to be used and developed in the treatment of premature infants.

\section{Use of touch in therapeutic interventions}

Therapeutic touch interventions such as those described above have been demonstrated to be highly effective in improving outcomes for very premature infants or infants with other perinatal obstacles to development. In older children, sensory-based interventions have become a prominent clinical focus for a variety of NDDs. Sensory integration therapy (SIT) addresses hypo- or hyperresponsiveness to sensory input using child-directed, one-on-one play between the therapist and the child, and is used frequently for children with ASD and disorders of scholastic skill acquisition. SIT typically involves a combination of sensory stimulation and movement, or a sensory stimulus to which the child is asked to respond. The therapist guides the child through a series of activities incorporating these elements in a way that is simultaneously challenging and fun (Ayres 1979). A theoretical developmental hierarchy for sensory systems provides the framework for SIT. In this schema, sensory, cognitive, and behavioral systems are represented as a pyramid, the base of which comprises the tactile, vestibular and proprioceptive systems. Distal senses such as vision and audition build upon this level, and more complex sensorimotor, behavioral, and cognitive 
functions represent the highest levels of this hierarchy (Williams and Shellenberger 1996). Thus tactile, proprioceptive, and vestibular sensation (i.e., proximal senses) are considered critical prerequisites for the proper development and integration of other senses as they are earliest to develop and provide a physical framework of the child's body in space and its boundaries upon which more distal senses such as vision and audition, and later more complex cognitive and behavioral skills, build. There have been conflicting reports as to the efficacy of SIT in various NDD populations (Hoehn and Baumeister 1994; Dawson and Watling 2000; Baranek 2002; Roberts et al. 2007), although there is a consensus that the approach is under-studied and more extensive and rigorous research is needed (Baranek 2002; Schaaf and Miller 2005).

Other therapeutic approaches that target the tactile system specifically include repetitive brushing of the skin and the use of deep pressure stimulation with weighted vests. Weighted vests are posited to improve attention (Fertel-Daly et al. 2001) and hyperactivity, possibly by modulating arousal levels. Olson and Moulton (2004) collected reports from practitioners using weighted vests, and found that therapists did endorse improvements in attention, although a self-selecting response bias in this approach was acknowledged. A review conducted by Stephenson and Carter (2009) found little empirical evidence for the efficacy of weighted vests.

A more controversial approach is the Wilbarger protocol, an intensive home treatment program in which deep pressure massage using a brush and gentle joint compression are administered by parents several times a day, often prescribed as part of a "sensory diet" (Wilbarger and Wilbarger 1991) to ameliorate sensory defensiveness and enhance attention. Enthusiastic anecdotal evidence suggests efficacy in these areas, but very little empirical research has been conducted. Kimball and colleagues (2007) measured salivary cortisol levels in a small group of four children before and after several administrations of the protocol, and found that levels changed significantly between pre- and post-test measurements. Although preliminary, this study provides the first experimental evidence that deep pressure stimulation affects arousal levels in these children.

The scarcity of empirical research for these popular therapeutic approaches targeting the somatosensory system illustrates the importance of collaborative, translational research. Basic scientists with expertise in characterizing neural and physiological responses and training in experimental design must join forces with clinicians and educators with expertise in behavior and real-life applications to effectively support these approaches with a rigorous evidence base, or to inform changes and improvements to current therapeutic techniques.

\section{Conclusions}

The studies included in this review reflect the central role of tactile processing in the development of social, communicative, and motor behavior. Disorders that affect neural development are associated with impairments in somatosensory discriminative abilities and affective responses to tactile stimulation, as measured by parent report, clinical observation, and psychophysical experiments. The neural mechanisms underlying these differences require further study, and may differ across NDDs, reflecting the different roles of tactile perception in motor control, social behavior, and autonomic arousal. Possible contributing systems include peripheral afferent pathology, particularly of the C-touch class of afferents, thalamocortical gating mechanisms that affect the propagation and modulation of tactile information to the cortex, cortical structural patterns such as columnar organization and GABA-mediated lateral inhibition, intracortical oscillatory patterns, and top-down modulation of sensory cortical responses by prefrontal or other areas of cortex devoted to cognitive control.

NDDs that have historically been attributed solely to motor system dysregulation, such as $\mathrm{CP}$, are now being re-examined and the importance of the somatosensory system has become clear. The lack of tactile input for neonates has profound negative consequences, not just for sensory processing, but also for development more generally. These effects can be ameliorated by therapeutic tactile input for infants such as massage and kangaroo care. Especially for NDDs of complex or unknown genetic origin such as ADHD and ASD, tactilecentered therapeutic approaches in older children may be effective in modulating arousal, attention, and sensory defensiveness, but there is a pressing need for translational research both on the efficacy of these approaches and their mechanistic rationales.

Acknowledgments The author thanks Dr. Grace Baranek, Dr. Francis McGlone, and Jennifer H. Foss-Feig for helpful comments on the manuscript.

\section{References}

Ayres AJ. Sensory integration and the child. Los Angeles: Western Psychological Services; 1979.

Baranek GT. Efficacy of sensory and motor interventions for children with autism. J Autism Dev Disord. 2002;32(5):397-422.

Baranek GT, Foster LG, Berkson G. Tactile defensiveness and stereotyped behaviors. Am J Occup Ther. 1997;51(2):91-5.

Baranek GT, Roberts JE, David FJ, Sideris J, Mirrett PL, Hatton DD, et al. Developmental trajectories and correlates of sensory processing in young boys with fragile $\mathrm{X}$ syndrome. Phys Occup Ther Pediatr. 2008;28(1):79-98.

Blakemore SJ, Tavassoli T, Calo S, Thomas RM, Catmur C, Frith U, et al. Tactile sensitivity in Asperger syndrome. Brain Cogn. 2006;61:5-13. 
Bröring T, Rommelse N, Sergeant J, Scherder E. Sex differences in tactile defensiveness in children with ADHD and their siblings. Dev Med Child Neurol. 2008;50(2):129-33.

Bureau I, Shepard GMG, Svoboda K. Circuit and plasticity defects in developing somatosensory cortex of fmr1 knock-out mice. J Neurosci. 2008;28(20):5178-88.

Carmona S, Villarroya O, Bielsa A, Tremols V, Soliva JC, Rovira M, et al. Global and regional grey matter reductions in ADHD: A voxelbased morphometric study. Neurosci Lett. 2005;389(2):88-93.

Carvell GE, Simons DJ. Abnormal tactile experience early in life disrupts active touch. J Neurosci. 1996;16(8):2750-7.

Cascio CJ, McGlone F, Folger S, Tannan V, Baranek G, Pelphrey KA, et al. Tactile perception in adults with autism: a multidimensional psychophysical study. J Autism Det Disord. 2008;38:127-37.

Case-Smith J. The relationships among sensorimotor components, fine motor skill, and functional performance in preschool children. Am J Occup Ther. 1995;49(7):645-52.

Casler L. The effects of extra tactile stimulation on a group of institutionalized infants. Genet Psychol Monogr. 1965;71:137-75.

Cauller L. Layer I of primary sensory neocortex: where top-down converges upon bottom-up. Behav Brain Res. 1995;71:163-70.

Cermak SA, Daunhauer LA. Sensory processing in the postinstitutionalized child. Am J Occup Ther. 1997;5:500-7.

Cermak SA, Groza V. Sensory processing problems in postinstitutionalized children: implications for social work. Child Adolesc Soc Work J. 2004;15(1):5-37.

Clayton K, Fleming JM, Copley J. Behavioral responses to tactile stimuli in children with cerebral palsy. Phys Occup Ther Pediatr. 2003;23(1):43-62.

Cooper J, Majnemer A, Rosenblatt B. The determination of sensory deficits in children with hemiplegic cerebral palsy. J Child Neurol. 1995;10:300-9.

Corbetta D, Snap-Childs W. Seeing and touching: the role of sensorymotor experience on the development of infant reaching. Infant Behav Dev. 2009;32:44-58.

Coskun MA, Varghese L, Reddoch S, Castillo EM, Pearson DA, Loveland KA, et al. How somatic cortical maps differ in autistic and typical brains. NeuroReport. 2009;20(2):175-9.

Curry J, Exner C. Comparison of tactile preferences in children with and without cerebral palsy. Am J Occup Ther. 1988;42(6):371-7.

Dahlin LB, Komoto-Tufvesson Y, Sälgeback S. Surgery of the spastic hand in cerebral palsy. Improvement in stereognosis and hand function after surgery. J Hand Surg. 1998;23:334-9.

Dawson G, Watling R. Interventions to facilitate auditory, visual, and motor integration in autism: a review of the evidence. J Autism Dev Disord. 2000;30(5):415-21.

Dockstader C, Gaetz W, Cheyne D, Wang F, Castellanos FX, Tannock R. MEG event-related desynchronization and synchronization deficits during basic somatosensory processing in individuals with ADHD. Behav Brain Funct. 2008;4(8):1-13.

Dockstader C, Gaetz W, Cheyne D, Tannock R. Abnormal neural reactivity to unpredictable sensory events in attention-deficit/ hyperactivity disorder. Biol Psychiatry. 2009;66(4):376-83.

Dunbar R. Grooming, gossip, and the evolution of language. Cambridge: Harvard University Press; 1996.

Ferber SG, Feldman R, Makhoul IR. The development of maternal touch across the first year of life. Early Hum Dev. 2008;24:36370.

Fertel-Daly D, Bedell G, Hinojosa J. Effects of a weighted vest on attention to task and self-stimulatory behaviors in preschoolers with pervasive developmental disorders. Am J Occup Ther. 2001;55:629-40.

Field D, Garland M, Williams K. Correlates of specific childhood feeding problems. J Paediatr Child Health. 2003;39:299-304.

Galvez R, Greenough WT. Sequence of abnormal dendritic spine development in primary somatosensory cortex of a mouse model of the fragile X mental retardation syndrome. Am J Med Genet. 2005;135(2):155-60.

Galvez R, Gopal AR, Greenough WT. Somatosensory cortical barrel dendritic abnormalities in a mouse model of the fragile $\mathrm{X}$ mental retardation syndrome. Brain Res. 2003;971(1):83-9.

Ghanizadeh A. Tactile sensory dysfunction in children with ADHD. Behav Neurol. 2008;20(3-4):107-12.

Gick B, Jóhannsdóttir KM, Gibraiel D, Mühlbauer J. Tactile enhancement of auditory and visual speech perception in untrained perceivers. J Acoust Soc Am. 2008;123:EL72-76.

Grandin T. An inside view of autism. Fort Collins: Colorado State University; 2000.

Greenough WT, Klintsova AY, Irwin SA, Galvez R, Bates KE, Weiler IJ. Synaptic regulation of protein synthesis and the fragile $\mathrm{X}$ protein. Proc Natl Acad Sci. 2001;98(13):7101-6.

Güçlü B, Tanidir C, Mukaddes NM, Unal F. Tactile sensitivity of normal and autistic children. Somatosens Motor Res. 2007;24: 21-33.

Harlow HF, Harlow MK. The effect of rearing conditions on behavior. Bull Menninger Clin. 1962;26:213-24.

Harris G. Development of taste and food preferences in children. Curr Opin Clin Nutr Metab Care. 2008;11:315-9.

Hertenstein MJ, Verkamp JM, Kerestes AM, Homes RM. The communicative function of touch in humans, nonhuman primates, and rats: a review and synthesis of the empirical research. Genet Soc Gen Psychol Monogr. 2006;132:5-94.

Hoehn TP, Baumeister AA. A critique of the application of sensory integration therapy to children with learning disabilities. J Learn Disabil. 1994;27:338-50.

Hoon AH, Stashinko EE, Nage LM, Lin DD, Keller J, Bastian A, et al. Sensory and motor deficits in children with cerebral palsy born preterm correlate with diffusion tensor imaging abnormalities in thalmocortical pathways. Dev Med Child Neurol. 2009;51 (9):697-704

Jean AD, Stack DM. Functions of maternal touch and infants' affect during face-to-face interactions: new directions for the still-face. Infant Behav Dev. 2009;32:123-8.

Kimball JG, Lynch KM, Stewart KC, Williams NE, Thomas MA, Atwood KD. Using salivary cortisol to measure the effects of a Wilbarger protocol based procedure on sympathetic arousal: A pilot study. Am J Occup Ther. 2007;61(4):406-13.

Kuhn CM, Schanberg SM. Responses to maternal separation: mechanisms and mediators. Int J Dev Neurosci. 1998;16(3-4):261-70.

Kulak W, Sobaniec W, Kuzia SJ, Bockowski L. Neurphysiologic and neuroimaging studies of brain plasticity in children with spastic cerebral palsy. Exp Neurol. 2006;198(1):4-11.

Kunesch E, Binkofski F, Freund HJ. Invariant temporal characteristics of manipulative hand movements. Exp Brain Res. 1989;78 (3):539-46.

Lesny I, Stehlík A, Tomásek J, Tománková A, Havlícek I. Sensory disorders in cerebral palsy: two point discrimination. Dev Med Child Neurol. 1993;35(5):402-5.

Lin SH, Cermak S, Coster WJ, Miller L. The relation between length of institutionalization and sensory integration in children adopted from Eastern Europe. Am J Occup Ther. 2005;59(2):139-47.

Liu Q, Vrontou S, Rice F, Zylka MJ, Dong X, Anderson DJ Molecular genetic visualization of a rare subset of unmyelinated sensory neurons that may detect gentle touch. Nat Neurosci. 2007;10:946-8.

Löken LS, Wessberg J, Morrison I, McGlone F, Olausson H. Coding of pleasant touch by unmyelinated afferents in humans. Nat Neurosci. 2009;12:547-8.

Main M, Stadtman J. Infant response to rejection of physical contact by the mother: aggression, avoidance, and conflict. J Am Acad Child Psych. 1981;20:292-307. 
McGlone F, Vallbo AB, Olausson H, Loken LS, Wessberg J. Discriminative touch and emotional touch. Can J Exp Psychol. 2007;61:171-83.

Metcalfe JS, McDowell K, Chang TY, Chen LC, Jeka JJ, Clark JE. Development of somatosensory-motor integration: and eventrelated analysis of infant posture in the first year of independent walking. Dev Psychobiol. 2005;46:19-35.

Miyazaki M, Fujii E, Saijo T, Mori K, Hashimoto T, Kagami S, Kuroda Y. Short-latency somatosensory evoked potentials in infantile autism: evidence of hyperactivity in the right primary somatosensory area. Dev Med Child Neurol. 2007;49:13-7.

Montagu A. Touching: the human significance of the skin. New York: Perennial Library; 1986.

Moszkowski RJ, Stack DM, Chiarella SS. Infant touch with gaze and affective behaviors during mother-infant still-face interactions: co-occurrence and functions of touch. Infant Behav Dev. 2009;32:392-403.

Mottron L, Dawson M, Soulières I, Hubert B, Burack J. Enhanced perceptual functioning in autism: an update, and eight principles of autistic perception. J Autism Dev Disord. 2006;36 (1):27-43.

Muir DW. Adult communications with infants through touch: the forgotten sense. Hum Dev. 2002;45:95-9.

Myers BJ. Mother-infant bonding: the status of the critical-period hypothesis. Dev Rev. 1984;4:240-74.

Olausson H, Lamarre Y, Backlund H, Morin C, Wallin BG, Starck G, et al. Unmyelinated tactile afferents signal touch and project to insular cortex. Nat Neurosci. 2002;5:900-4.

Olson LJ, Moulton HJ. Use of weighted vests in pediatric occupational therapy practice. Phys Occup Ther Pediatr. 2004;24(3):45-60.

O'Riordan M, Passetti F. Discrimination in autism within different sensory modalities. J Autism Dev Disord. 2006;36:665-75.

Parush S, Sohmer H, Steinberg A, Kaitz M. Somatosensory functioning in children with attention deficit hyperactivity disorder. Dev Med Child Neurol. 1997;39(7):464-8.

Parush S, Sohmer H, Steinberg A, Kaitz M. Somatosensory function in boys with ADHD and tactile defensiveness. Physiol Behav. 2007;90(4):553-8.

Pernon E, Pry R, Baghdadli A. Autism: tactile perception and emotion. J Intellect Disabil Res. 2007;51(8):580-7.

Pihko E, Lauronen L. Somatosensory processing in healthy newborns. Exp Neurol. 2004;190 Suppl 1:S2-7.

Porter RH. The biological significante of skin-to-skin contact and maternal odours. Acta Paediatr. 2004;93(12):1560-2.

Restivo L, Ferrari F, Passino E, Sgobio C, Bock J, Oostra BA, et al. Enriched environment promotes behavioral and morphological recovery in a mouse model for the fragile $\mathrm{X}$ syndrome. Proc Natl Acad Sci. 2005;102(32):11557-62.

Reynolds S, Lane SJ. Sensory overresponsivity in and anxiety in children with ADHD. Am J Occup Ther. 2009;63(4):433-40.

Roberts JE, King-Thomas L, Boccia ML. Behavioral indexes of efficacy sensory integration therapy. Am J Occup Ther. 2007;61 (5):555-62.

Rogers SJ, Hepburn S, Wehner E. Parent reports of sensory symptoms in toddlers with autism and those with other developmental disorders. J Autism Dev Disord. 2003;33(6):631-42.

Sanger TD, Kukke SN. Abnormalities of tactile sensory function in children with dystonic and diplegic cerebral palsy. J Child Neurol. 2007;22:289-93.

Schaaf RC, Miller LJ. Occupational therapy using a sensory integrative approach for children with developmental disabilities. Ment Retard Dev Disabil Res Rev. 2005;11(2):143-8.

Schanberg SM, Field TM. Sensory deprivation stress and supplemental stimulation in the rat pup and preterm human neonate. Child Dev. 1987;58(6):1431-47.
Schanberg SM, Ingledue VF, Lee JY, Hannun YA, Batolome JV. PKC alpha mediates maternal touch regulation of growth-related gene expression in infant rats. Neuropsychopharmacology. 2003;28 (6):1026-30

Scherder EJA, Rommelse NNJ, Bröring T, Faraone SV, Sergeant JA. Somatosensory functioning and experienced pain in ADHD families: a pilot study. Eur J Paediatr Neurol. 2008;12(6):461-9.

Selby L, Zhang C, Sun Q. Major defects in neocortical GABAergic inhibitory circuits in mice lacking the fragile $\mathrm{X}$ mental retardation protein. Neurosci Lett. 2007;412(3):227-32.

Shochat T, Tzischinsky O, Engel-Yeger B. Sensory hypersensitivity as a contributing factor in the relation between sleep and behavioral disorders in normal schoolchildren. Behav Sleep Med. 2009;7: 53-62.

Soechting JF, Flanders M. Sensorimotor control of contact force. Curr Opin Neurobiol. 2008;18:565-72.

Sorensen ER. Early tactile communication and the patterning of human organization: a New Guinea case study. In: Bullowa M, editor. Before speech: the beginning of interpersonal communication. Cambridge: Cambridge University Press; 1979. p. 289-306.

Stack DM, Muir DW. Adult tactile stimulation during face-to-face interactions modulates five-month-olds' affect and attention. Child Dev. 1992;63(6):1509-25.

Stephenson J, Carter M. The use of weighted vests with children with autism spectrum disorders and other disabilities. J Autism Dev Disord. 2009;39(1):105-14.

Tomchek SD, Dunn W. Sensory processing in children with and without autism: a comparative study using the Short Sensory Profile. Am J Occup Ther. 2007;61(2):190-200.

Tomita Y, Fukuda C, Kafo Y, Maegaki Y, Shiota S, Amisaki T. Topographic MN-SSEPs (N18, N20, and N30) might characterize underlying CNS involvements in representative types of cerebral palsy. Brain Dev. 2006;28(10):653-9.

Tommerdahl M, Tannan V, Cascio CJ, Baranek GT, Whitsel BL. Vibrotactile adaptation fails to enhance spatial localization in adults with autism. Brain Res. 2007;1154:116-23.

Underdown A, Barlow J, Chung V, Stewart-Brown S. Massage intervention for promoting mental and physical health in infants aged under 6 months. Cochrane Database Syst Rev. 2006;18: CD005038.

Vallbo $\mathrm{AB}$, Olausson $\mathrm{H}$, Wessberg J. Unmyelinated afferents constitute a second system coding tactile stimuli of the human hairy skin. J Neurophysiol. 1999;81(6):2753-63.

Van Heest AE, House J, Putnam M. Sensibility deficiencies in the hands of children with spastic hemiplegia. J Hand Surg. 1993;18 (2):278-81.

Weiss SJ, Wilson P, Hertenstein MJ, Campos RG. The tactile context of a mother's caregiving implications for attachment of low birth weight infants. Infant Behav Dev. 2000;23:91-111.

Wilbarger P, Wilbarger J. Sensory defensiveness in children ages 2 12: an intervention guide for parents and other caretakers. Santa Barbara: Avanti Educational Programs; 1991.

Wilke M, Staudt M. Does damage to somatosensory circuits underlie motor impairment in cerebral palsy? Dev Med Child Neurol. 2009;51(9):686-7.

Williams MS, Shellenberger S. How does your engine run? A Leader's guide to the alert program for self-regulation. Albuquerque: TherapyWorks, Inc; 1996.

Wingert JR, Burton H, Sinclair RJ, Brunstrom JE, Damiano DL. Tactile sensory abilities in cerebral palsy: deficits in roughness and object discrimination. Dev Med Child Neurol. 2008;50(11):832-8.

Yochman A, Parush S, Ornoy A. Responses of preschool children with and without ADHD to sensory events in daily life. Am J Occup Ther. 2004;58:294-302. 